\title{
Improving WPM2 for (Weighted) Partial MaxSAT *
}

\author{
Carlos Ansótegui $^{1}$, Maria Luisa Bonet ${ }^{2}$, Joel Gabàs ${ }^{1}$, and Jordi Levy ${ }^{3}$ \\ 1 DIEI, Univ. de Lleida \\ carlos@diei.udl.cat \\ joel.gabas@diei.udl.cat \\ 2 LSI, UPC \\ bonet@lsi.upc.edu \\ 3 IIIA-CSIC \\ levy@iiia.csic.es
}

\begin{abstract}
Weighted Partial MaxSAT (WPMS) is an optimization variant of the Satisfiability (SAT) problem. Several combinatorial optimization problems can be translated into WPMS. In this paper we extend the state-of-the-art WPM2 algorithm by adding several improvements, and implement it on top of an SMT solver. In particular, we show that by focusing search on solving to optimality subformulas of the original WPMS instance we increase the efficiency of WPM2. From the experimental evaluation we conducted on the PMS and WPMS instances at the 2012 MaxSAT Evaluation, we can conclude that the new approach is both the best performing for industrial instances, and for the union of industrial and crafted instances.
\end{abstract}

\section{Introduction}

In the last decade Satisfiability (SAT) solvers have progressed dramatically in performance due to new algorithms, such as, conflict directed clause learning [36], and better implementation techniques. Thanks to these advances, nowadays the best SAT solvers can tackle hard decision problems. Our aim is to push this technology forward to deal with optimization problems.

The Maximum Satisfiability (MaxSAT) problem is the optimization version of SAT. The idea behind this formalism is that sometimes not all constraints of a problem can be satisfied, and we try to satisfy the maximum number of them. The MaxSAT problem can be further generalized to the Weighted Partial MaxSAT (WPMS) problem.

In the MaxSAT community, we find two main classes of algorithms: branch and bound $[17,22,24,26,27]$ and SAT-based [2,14,19-21,31-33]. The latter clearly dominate on industrial and some crafted instances, as we can see in the results of the last 2012 MaxSAT Evaluation. SAT-based MaxSAT algorithms

\footnotetext{
* This research has been partially founded by the CICYT research projects TASSAT
} (TIN2010-20967-C04-01/03/04) and ARINF (TIN2009-14704-C03-01). 
basically reformulate a MaxSAT instance into a sequence of SAT instances. By solving these SAT instances the MaxSAT problem can be solved [6].

In this paper we revisit the SAT-based MaxSAT algorithm WPM2 [5] which belongs to a family of algorithms that exploit the information from the unsatisfiable cores the underlying SAT solver provides. This algorithm is the natural extension to the weighted case of the Partial MaxSAT algorithm PM2 $[4,3]$. In our experimental investigation the original WPM2 algorithm solves 796 out of 1474 from the whole benchmark of PMS and WPMS industrial and crafted instances at the 2012 MaxSAT Evaluation. We have extended WPM2 with several complementary improvements. First of all, we apply the stratification approach described in [2], what results in solving 74 additional instances. Secondly, we introduce a new criteria to decide when soft clauses can be hardened, that provides 66 additional solved instances. The hardening of soft clauses in MaxSAT SAT-based solvers has been previously studied in [2, 33]. Finally, our most effective contribution is to introduce a new strategy that focuses search on solving to optimality subformulas of the original MaxSAT instance. Actually, the new WPM2 algorithm is parametric on the approach we use to optimize these subformulas. This allows to combine the strength of exploiting the information extracted from unsatisfiable cores and other optimization approaches. By solving these smaller optimization problems we get the most significant boost in our new WPM2 algorithm. In particular, we experiment with three approaches: (i) refine the lower bound on these subformulas with the subsetsum function $[13,5]$, (ii) refine the upper bound with the strategy applied in minisat+ [15], SAT4J [10], qmaxsat [21] or ShinMaxSat [20], and (iii) a binary search scheme where the lower bound and upper bound are refined as in the previous approaches. The best performing approach in our experimental analysis is the second one and it allows to solve up to 238 additional instances. As a summary, the overall speed-up we achieved on the original WPM2 solver is about 378 additional solved instances, a $47 \%$ more.

As we mentioned, SAT-based MaxSAT algorithms reformulate a MaxSAT instances into a sequence of SAT instances. Obviously, it is important to use an efficient SAT solver. Also, most SAT-based MaxSAT algorithms require the addition of Pseudo-Boolean (PB) linear constraints as a result of the reformulation process. These $\mathrm{PB}$ constraints are used to bound the cost of the optimal assignment. Currently, in most state-of-the-art SAT-based MaxSAT solvers, PB constraints are translated into SAT. However, there is no known SAT encoding which can guarantee the original propagation power of the constraint, i.e, what we call arc-consistency, while keeping the translation low in size. The best approach so far, has a cubic complexity [8]. This can be a bottleneck for WPM2 [5] and also for other algorithms such as, BINCD [19] or SAT4J [10].

In order to treat $\mathrm{PB}$ constraints with specialized inference mechanisms and a moderate cost in size, while preserving the strength of SAT techniques for the rest of the formula, we use the Satisfiability Modulo Theories (SMT) technology [35]. Related work in this sense can be found in [34]. Also, in [1] a Weighted Constraint 
Satisfaction Problems (WCSP) solver implementing the original WPM1 [4] algorithm is presented.

An SMT instance is a generalization of a Boolean formula in which some propositional variables have been replaced by predicates with predefined interpretations from background theories such as, e.g., linear integer arithmetic. Most modern SMT solvers integrate a SAT solver with decision procedures (theory solvers) for sets of literals belonging to each theory. This way, we can hopefully get the best of both worlds: in particular, the efficiency of the SAT solver for the Boolean reasoning and the efficiency of special-purpose algorithms for the theory reasoning.

Another reasonable choice would be to use a PB solver, which can be seen as a particular case of an SMT solver specialized on the theory of PB constraints [28, 29]. However, if we also want to solve problems modeled with richer formalisms like WCSP, the SMT approach seems a better choice since we can take advantage of a wide range of theories [1].

In this work, we implemented both the last version of the WPM1 algorithm [2] and the revisited version of the WPM2 algorithm on top the of the SMT solver Yices. Then, we performed an extensive experimental evaluation comparing them with the best two solvers for PMS and WPMS categories at the 2012 MaxSAT Evaluation and with three additional solvers that did not take part but have been reported to exhibit good performance: bincd2, which is the new version of the BINCD algorithm [19] described in [33], with the best configuration reported by authors, maxhs from [14], which consists in an hybrid SAT and Integer Linear Programming (ILP) approach, and ilp which performs a translation of WPMS into ILP solved with IBM-CPLEX studio124 [7].

We observe that the implementation on SMT of our new WPM2 algorithm with the second approach for optimizing the subformulas is the best performing solver for both PMS and WPMS industrial instances. We also observe that it is the best performing for the union of PMS and WPMS industrial and crafted instances, what shows this is a robust approach. These results make us conjecture that by improving the interaction of our new WPM2 algorithm with diverse optimization techniques applied on the subformulas we can get additional speedups.

This paper proceeds as follows. Section 2 presents some preliminary concepts. Section 3 describes WPM2 [5] and the new improvements. Section 4 describes the SMT problem and discuss some implementation details of the SMT-based MaxSAT algorithms. Section 5 presents the experimental evaluation. Finally, Section 6 shows the conclusions and the future work.

\section{Preliminaries}

A literal is either a Boolean variable $x$ or its negation $\bar{x}$. A clause $C$ is a disjunction of literals. A weighted clause is a pair $(C, w)$, where $C$ is a clause and $w$ is a natural number or infinity, indicating the penalty for falsifying the 
clause $C$. A Weighted Partial MaxSAT formula is a multiset of weighted clauses

$$
\varphi=\left\{\left(C_{1}, w_{1}\right), \ldots,\left(C_{m}, w_{m}\right),\left(C_{m+1}, \infty\right), \ldots,\left(C_{m+m^{\prime}}, \infty\right)\right\}
$$

where the first $m$ clauses are soft and the last $m^{\prime}$ clauses are hard. The set of variables occurring in a formula $\varphi$ is noted $\operatorname{as} \operatorname{var}(\varphi)$.

A (total) truth assignment for a formula $\varphi$ is a function $I: \operatorname{var}(\varphi) \rightarrow\{0,1\}$, that can be extended to literals, clauses and SAT formulas. For MaxSAT formulas is defined as $I\left(\left\{\left(C_{1}, w_{1}\right), \ldots,\left(C_{m}, w_{m}\right)\right\}\right)=\sum_{i=1}^{m} w_{i}\left(1-I\left(C_{i}\right)\right)$. The optimal cost of a formula is $\operatorname{cost}(\varphi)=\min \{I(\varphi) \mid I: \operatorname{var}(\varphi) \rightarrow\{0,1\}\}$ and an optimal assignment is an assignment $I$ such that $I(\varphi)=\operatorname{cost}(\varphi)$.

The Weighted Partial MaxSAT problem for a Weighted Partial MaxSAT formula $\varphi$ is the problem of finding an optimal assignment.

\section{WPM2 algorithm}

The WPM2 algorithm [5] is described in Algorithm 1. The fragments in gray (lines 4, 10, 11, 13- 18 and 20) correspond to the new improvements we have incorporated.

In the WPM2 algorithm, we extend soft clauses $C_{i}$ with a unique fresh auxiliary blocking variable $b_{i}$ obtaining $\varphi_{w}=\left\{C_{i} \vee b_{i}\right\}_{i=1 \ldots m} \cup\left\{C_{m+i}\right\}_{i=1 \ldots m^{\prime}}$. Notice that $b_{i}$ will be set to true by a SAT solver on $\varphi_{w}$ if $C_{i}$ is false. We also work with a set $A L$ of at-least PB constraints of the form $\sum_{i \in A} w_{i} b_{i} \geq k$ on the variables $b_{i}$, and a similar set $A M$ of at-most constraints of the form $\sum_{i \in A} w_{i} b_{i} \leq k$, that are modified at every iteration of the algorithm.

Intuitively, the WPM2 algorithm refines at every iteration the lower bound on $\varphi$ till it reaches the optimum $\operatorname{cost}(\varphi)$. The $A M$ constraints are used to bound the cost of the falsified clauses. The $A L$ constraints are used to impose that subsets of soft clauses have a minimum cost and to compute the $A M$ constraints, as we will see later. The algorithm ends when $\varphi_{w} \cup C N F(A L \cup A M)$ becomes satisfiable ${ }^{4}$, where CNF is the translation to SAT of the PB constraints.

Technically speaking, the $A L$ constraints give lower bounds on $\operatorname{cost}(\varphi)$. The $A M$ constraints enforce that all solutions of the set of constraints $A L \cup A M$ are the solutions of $A L$ of minimal cost. This ensures that any solution of the formula sent to the solver, $\varphi_{w} \cup C N F(A L \cup A M)$, if there is any, is an optimal assignment of $\varphi$. Therefore, given a set of at-least constraints $A L$ we compute a corresponding set of at-most constraints $A M$ as follows. First, we need to introduce the notion of core and cover. A core is a set of indexes $A$ such that $\sum_{i \in A} w_{i} b_{i} \geq k \in A L$. Function $\operatorname{core}\left(\sum_{i \in A} w_{i} b_{i} \geq k\right)$ returns the core $A$ and function cores $(A L)$ returns $\{\operatorname{core}(a l) \mid a l \in A L\}$. Covers are defined from cores as follows.

Definition 1. Given a set of cores $L$, we say that the set of indexes $A$ is a cover of $L$, if it is a minimal non-empty set such that, for every $A^{\prime} \in L$, if $A^{\prime} \cap A \neq \emptyset$, then $A^{\prime} \subseteq A$. Given a set of cores $L$, we denote the set of covers of $L$ as $\operatorname{SC}(L)$.

\footnotetext{
${ }^{4}$ The $A L$ constraints are redundant, i.e., not required to be sent to the SAT solver for the soundness of the algorithm but help to speed up the search.
} 


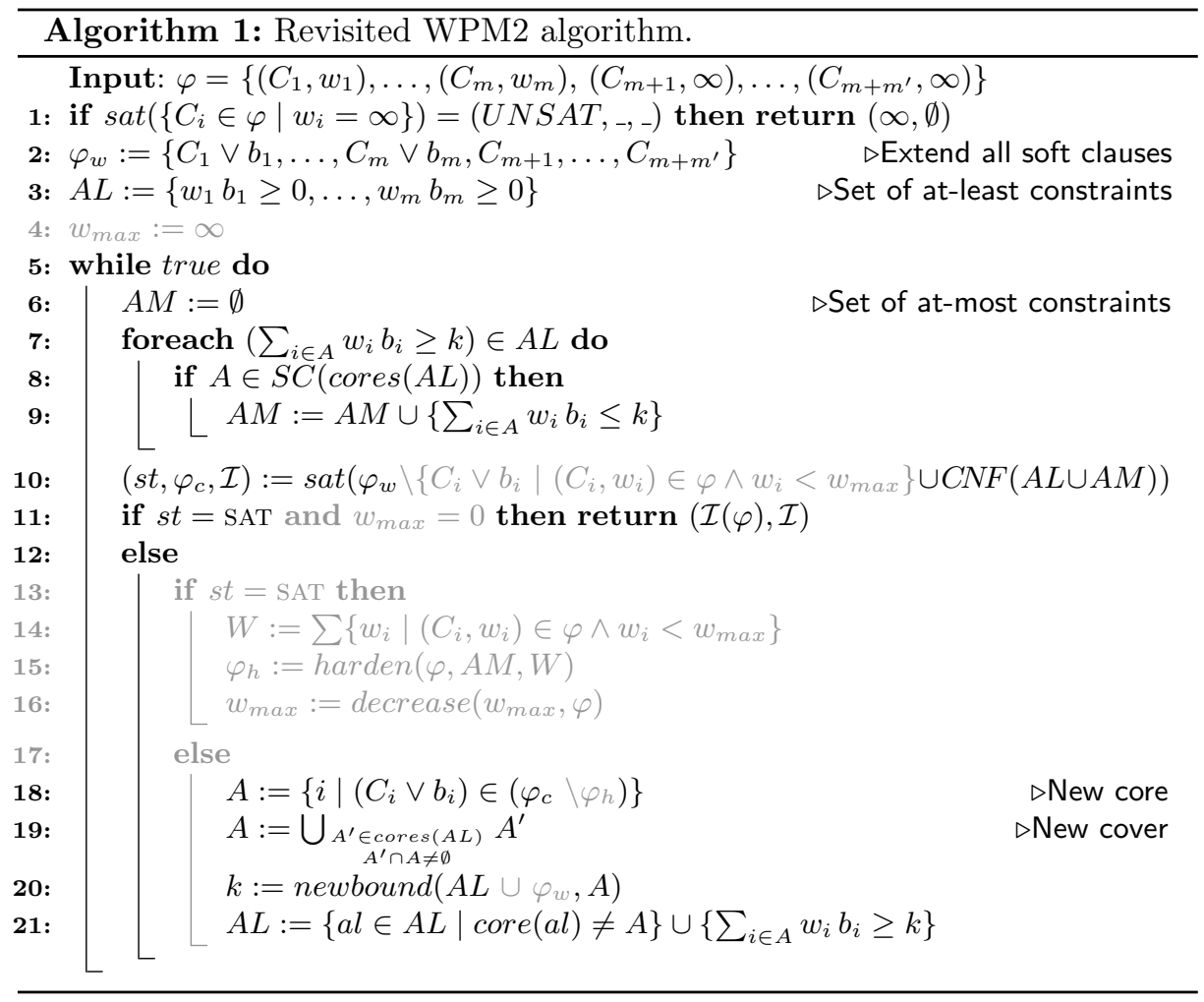

Given a set $A L$, the set $A M$ is the set of at-most constrains $\sum_{i \in A} w_{i} b_{i} \leq k$ such that $A \in S C(\operatorname{cores}(A L))$ and $k$ is the solution of minimizing $\sum_{i \in A} w_{i} b_{i}$ subject to $A L$ and $b_{i} \in\{0,1\}$.

The algorithm starts with $A L=\left\{w_{1} b_{1} \geq 0, \ldots, w_{m} b_{m} \geq 0\right\}$ and the corresponding $A M:=\left\{w_{1} b_{1} \leq 0, \ldots, w_{m} b_{m} \leq 0\right\}$ that ensures that the unique solution of $A L \cup A M$ is $b_{1}=\cdots=b_{m}=0$ with cost $0{ }^{5}$. At every iteration, the algorithm calls a SAT solver with $\varphi_{w} \cup C N F(A L \cup A M)$. If it returns SAT, then the interpretation $\mathcal{I}$ is a MaxSAT solution of $\varphi$ and we return the optimal $\operatorname{cost} \mathcal{I}(\varphi)$. If it returns UNSAT, then we use the information of the unsatisfiable core $\varphi_{c}$ obtained by the SAT solver to enlarge the set $A L$, excluding more interpretations on the $b_{i}$ 's that are not partial solutions of $\varphi_{w}$. Before calling again the SAT solver, we update $A M$ conveniently, to ensure that solutions to the new constraints $A L \cup A M$ are still minimal solutions of the new $A L$ constraint set. Notice that in every iteration the set of solutions of $\left\{b_{1}, \ldots, b_{m}\right\}$ defined by $A L$ is decreased, whereas the set of solutions of $A M$ is increased.

\footnotetext{
${ }^{5}$ In the implementation, we do not add a blocking variable to a soft clause till it appears into a core.
} 
One key point in WPM2 is to compute the newbound $(A L, A)$ (line 20) which corresponds to the following optimization problem:

$$
\operatorname{minimize} \sum_{i \in A} w_{i} \cdot b_{i} \quad \text { subject to }\left\{\sum_{i \in A} w_{i} \cdot b_{i} \geq k\right\} \cup A L
$$

where $k=1+\sum\left\{k^{\prime} \mid \sum_{i \in A^{\prime}} w_{i} b_{i} \leq k^{\prime} \in A M \wedge A^{\prime} \subseteq A\right\}$.

Notice that by removing the $A L$ constraints in (1), we get the subsetsum problem [13]. In the original WPM2 algorithm [5], the subsetsum problem is progressively solved until we get a solution that also satisfies the $A L$ constraints. This satisfiability check in the original WPM2 is performed with a SAT solver.

In what follows, we present how we have modified the original WPM2 algorithm (fragments in gray in Algorithm 1) by incorporating several improvements: the application of a stratified approach, the hardening of soft clauses and the optimization of the subformulas defined by the covers.

\subsection{Stratified Approach}

As in [4] for WPM1, we apply a stratified approach. The stratified approach (lines 4, 10, 11 and 16) consists in sending to the SAT solver only those soft clauses with weight $w_{i} \geq w_{\max }$. Then, when the SAT solver returns SAT, if there are still unsent clauses, we decrease $w_{\max }$ to include additional clauses to the formula. From [4], we also apply the diversity heuristic (line 16) which supplies us with an efficient method to calculate how we have to reduce the value of $w_{\max }$ in the stratified approach, so that, when there is a big variety of distinct weights, $w_{\max }$ decreases faster, and, when there is a low diversity, $w_{\max }$ is decreased to the following value of $w_{i}$. Similar approach with an alternative heuristic for grouping clauses can be found in [32].

\subsection{Clause Hardening}

The hardening of soft clauses in MaxSAT SAT-based solvers has been previously studied in $[11,25,23,18,30,2,33]$. Inspired by these works we study a hardening scheme for WPM2. While clause hardening was reported to have no positive effect in WPM1 [2], we will see that it boosts efficiency in WPM2.

The clause hardening (lines 14, 15 and 18) consists in considering hard those soft clauses whose satisfiability we know does not need to be reconsidered. We need some lemma ensuring that falsifying those soft clauses would lead us to suboptimal solutions. In the case of WPM1, all soft clauses satisfying $w_{i}>W$, where $W=\sum\left\{w_{i} \mid\left(C_{i}, w_{i}\right) \in \varphi \wedge w_{i}<w_{\max }\right\}$ is the sum of weights of clauses not sent to the SAT solver, can be hardened. The correctness of this transformation is ensured by the following lemma:

Lemma 1 (Lemma 24 in [6]).

Let $\varphi_{1}=\left\{\left(C_{1}, w_{1}\right), \ldots,\left(C_{m}, w_{m}\right),\left(C_{m+1}, \infty\right), \ldots,\left(C_{m+m^{\prime}}, \infty\right)\right\}$ be a MaxSAT formula with cost zero, let $\varphi_{2}=\left\{\left(C_{1}^{\prime}, w_{1}^{\prime}\right), \ldots,\left(C_{r}^{\prime}, w_{r}^{\prime}\right)\right\}$ be a MaxSAT 
formula without hard clauses and $W=\sum_{j=1}^{r} w_{j}^{\prime}$. Let

$$
\operatorname{harden}(w)= \begin{cases}w & \text { if } w \leq W \\ \infty & \text { if } w>W\end{cases}
$$

and $\varphi_{1}^{\prime}=\left\{\left(C_{i}, \operatorname{harden}\left(w_{i}\right)\right) \mid\left(C_{i}, w_{i}\right) \in \varphi_{1}\right\}$. Then, $\operatorname{cost}\left(\varphi_{1} \cup \varphi_{2}\right)=\operatorname{cost}\left(\varphi_{1}^{\prime} \cup \varphi_{2}\right)$, and any optimal assignment for $\varphi_{1}^{\prime} \cup \varphi_{2}$ is an optimal assignment of $\varphi_{1} \cup \varphi_{2}$.

However, this lemma is not useful in the case of WPM2 because we do not proceed by transforming the formula, like in WPM1. Therefore, we generalize this lemma. For this, we need to introduce the notion of optimal of a formula.

Definition 2. Given a MaxSAT formula $\varphi=\left\{\left(C_{1}, w_{1}\right), \ldots,\left(C_{m}, w_{m}\right)\right.$, $\left.\left(C_{m+1}, \infty\right), \ldots,\left(C_{m+m^{\prime}}, \infty\right)\right\}$, we say that $k$ is a (possible) optimal of $\varphi$ if there exists a subset $A \subseteq\{1, \ldots, m\}$ such that $\sum_{i \in A} w_{i}=k$.

Notice that, for any interpretation $I$ of the variables of $\varphi$, we have that $I(\varphi)$ is an optimal of $\varphi$. However, if $k$ is an optimal, there does not exist necessarily an interpretation $I$ satisfying $I(\varphi)=k$. Notice also that, given $\varphi$ and $k$, finding the next optimal, i.e. finding the smallest $k^{\prime}>k$ such that $k^{\prime}$ is an optimal of $\varphi$ is equivalent to the subset sum problem.

Lemma 2. Let $\varphi_{1} \cup \varphi_{2}$ be a MaxSAT formula and $k_{1}$ and $k_{2}$ values such that: $\operatorname{cost}\left(\varphi_{1} \cup \varphi_{2}\right)=k_{1}+k_{2}$ and any assignment $I$ satisfies $I\left(\varphi_{1}\right) \geq k_{1}$ and $I\left(\varphi_{2}\right) \geq k_{2}$. Let $k^{\prime}$ be the smallest possible optimal of $\varphi_{2}$ such that $k^{\prime}>k_{2}$. Let $\varphi_{3}$ be a set of soft clauses with $W=\sum\left\{w_{i} \mid\left(C_{i}, w_{i}\right) \in \varphi_{3}\right\}$.

Then, if $W<k^{\prime}-k_{2}$, then any optimal assignment $I^{\prime}$ of $\varphi_{1} \cup \varphi_{2} \cup \varphi_{3}$ assigns $I^{\prime}\left(\varphi_{2}\right)=k_{2}$

Proof. Let $I^{\prime}$ be any optimal assignment of $\varphi_{1} \cup \varphi_{2} \cup \varphi_{3}$. On the one hand, as for any other assignment, we have $I^{\prime}\left(\varphi_{2}\right) \geq k_{2}$.

On the other hand, any of the optimal assignments $I$ of $\varphi_{1} \cup \varphi_{2}$ can be extended (does not matter how) to the variables of $\operatorname{var}\left(\varphi_{3}\right) \backslash \operatorname{var}\left(\varphi_{1} \cup \varphi_{2}\right)$, such that

$$
I\left(\varphi_{1} \cup \varphi_{2} \cup \varphi_{3}\right)=I\left(\varphi_{1}\right)+I\left(\varphi_{2}\right)+I\left(\varphi_{3}\right) \leq k_{1}+k_{2}+W<k_{1}+k^{\prime}
$$

Now, assume that $I^{\prime}\left(\varphi_{2}\right) \neq k_{2}$, then $I^{\prime}\left(\varphi_{2}\right) \geq k^{\prime}$. As any other assignment, $I^{\prime}\left(\varphi_{1}\right) \geq k_{1}$. Hence, $I^{\prime}\left(\varphi_{1} \cup \varphi_{2} \cup \varphi_{3}\right) \geq k_{1}+k^{\prime}>I\left(\varphi_{1} \cup \varphi_{2} \cup \varphi_{3}\right)$, but this contradicts the optimality of $I^{\prime}$. Therefore, $I^{\prime}\left(\varphi_{2}\right)=k_{2}$.

In order to apply this lemma we have to consider partitions of the formula $\varphi_{1} \cup \varphi_{2}$ ensuring $\operatorname{cost}\left(\varphi_{1} \cup \varphi_{2}\right)=k_{1}+k_{2}$ and $I\left(\varphi_{1}\right) \geq k_{1}$ and $I\left(\varphi_{2}\right) \geq k_{2}$, for any assignment $I$. This can be easily ensured, in the case of WPM2, if both $\varphi_{1}$ and $\varphi_{2}$ are unions of covers. Then, we only have to check if the next possible optimal $k^{\prime}$ of $\varphi_{2}$ exceeds the previous one $k_{2}$ more than the sum $W$ of the weights of the clauses not sent to the SAT solver. In such a case, we can consider all soft 
clauses of $\varphi_{2}$ and their corresponding $A M$ constraint with $k_{2}$ as hard clauses. In other words, we do not need to recompute the partial optimal $k_{2}$ of $\varphi_{2}$.

Finally, in line 15 of Algorithm 1, function harden $(\varphi, A M, W)$ returns the set of soft clauses $\varphi_{h}$ that needs to be considered hard based on the previous analysis according to: the current set of covers $A M$, the next optimals of these covers and the sum of the weights $W$ of soft clauses beyond the current $w_{\max }$, i.e., not yet sent to the SAT solver.

\subsection{Cover Optimization}

As we have mentioned earlier, one key point in WPM2 is how to compute the newbound $(A L, A)$ (line 20). Actually, we can solve to optimality the subformulas defined by the union of the soft clauses related to the cover $A$ and the hard clauses.

Definition 3. Given a MaxSAT formula $\varphi=\left\{\left(C_{1}, w_{1}\right), \ldots,\left(C_{m}, w_{m}\right)\right.$, $\left.\left(C_{m+1}, \infty\right), \ldots,\left(C_{m+m^{\prime}}, \infty\right)\right\}$ and a set of indexes $A$, we define the subformula, $\varphi[A]$, as follows: $\left.\varphi[A]=\left\{\left(C_{i}, w_{i}\right) \in \varphi \mid i \in A \vee w_{i}=\infty\right)\right\}$

Solving to optimality $\varphi[A]$ give us the optimal value $k=\operatorname{cost}(\varphi[A])$ for the $A M$ constraint related to cover $A$. In order to do this, while taking advantage of the $A L$ constraints generated so far, we only have to extend the minimization problem corresponding to the newbound (1) function, by adding $\varphi_{w}$ to the constraints, i.e, newbound $\left(A L \cup \varphi_{w}, A\right)^{6}$. Notice that newbound $\left(A L \cup \varphi_{w}, A\right) \geq$ newbound $(A L, A)$.

In order to optimize $\varphi[A]$, we can use any exact approach related to MaxSAT, such as, MaxSAT branch and bound algorithms, MaxSAT SAT-based algorithms, saturation under the MaxSAT resolution rule, or we can use other solving techniques such as PB solvers or ILP techniques, etc. Our new WPM2 algorithm is parametric on any suitable optimization solving approach. In this work, we present three approaches.

The first and natural approach consists in iteratively refining (increasing) the lower bound on the optimal $k$ for $\varphi[A]$ by applying the subsetsum function as in the original WPM2. The procedure stops when we satisfy the constraints $A L \cup \varphi_{w}$. Notice that since we have included $\varphi_{w}$ into the set of constraints, the solution we will eventually get has to be optimal for $\varphi[A]$.

The second approach consists in iteratively refining (decreasing) the upper bound following the strategy applied in minisat+ [15], SAT4J [10], qmaxsat [21] or ShinMaxSat [20]. The upper bound $u b$ is initially set to the sum of the weights $w_{i}$ of the soft clauses in $\varphi[A]$. Then, we iteratively test whether $k=u b-1$ is feasible or not. Whenever we get a satisfying assignment, we update $u b$ to the sum of the weights $w_{i}$ of those soft clauses where $b_{i}$ evaluates to true under the satisfying assignment. If we get an unsatisfiable answer, the previous $u b$ is the optimal value for $\varphi[A]$.

\footnotetext{
${ }^{6}$ We can actually exclude from $\varphi_{w}$ all the soft clauses not in $\varphi[A]$.
} 
The third approach applies a binary search scheme $[19,12,16]$. We additionally refine the lower bound as in our first approach and the upper bound as in the second approach.

The worst case complexity, in terms of the number of calls to the SAT solver, of the new WPM2 algorithm is the number of times that the newbound function is called (bounded by the number of clauses) multiplied by the number of SAT calls needed in each call to the newbound function. This latter number is logarithmic on the sum of the weights of the clauses of the core if we use a binary search, hence essentially the number of clauses. Therefore, the worst case complexity, when using a binary search to solve to optimality the subformulas, is quadratic on the number of soft clauses.

In order to see that the number of calls to the newbound function is bounded by the number of clauses we just need to recall that WPM2 merges the covers. Consider a binary tree where the soft clauses are the leaves, and the internal nodes represent the merges (calls to the newbound function). A binary tree of $n$ leaves has n-1 internal nodes.

Solving to optimality all the covers can be very costly since these are NP-hard problems. Depending on the unsatisfiable cores we get in the general loop of the WPM2 algorithm some covers have to be merged. Therefore, we may argue that part of the work we did in order to optimize these covers can be useless ${ }^{7}$. For example, a reasonable strategy is to optimize the current cover only if it was not the result of merging other covers, i.e., when the last unsatisfiable core is contained into a cover. In the experimental evaluation, we will see that although the number of solved instances does not vary too much, the mean time for solving some families can be decreased.

\section{Engineering Efficient SMT-based MaxSAT Solvers}

We have implemented both the last version of the WPM1 algorithm [2] and the revisited version of the WPM2 algorithm on top the of the SMT solver Yices.

As we have said, an SMT instance is a generalization of SAT where some propositional variables are replaced by predicates with predefined interpretations from background theories. Among the theories considered in the SMT library [9] we are interested in QF_LIA (Quantifier-Free Linear Integer Arithmetic). With the QF_LIA theory we can model the PB constraints that SAT-based MaxSAT algorithms generate during their execution. Therefore, for the SMT-based MaxSAT algorithm, we just need to replace the conversion to CNF (line 10 in Algorithm 1) by the proper linear integer arithmetic predicates.

As suggested in $[16,31]$, we can preserve some learned lemmas from previous iterations that may help to reduce the search space. In order to do that, we execute the SMT solver in incremental mode. Within this mode, we can call the solve routine and add new clauses (assertions) on demand, while preserving learned lemmas. However, notice that our algorithms delete parts of the formula

\footnotetext{
7 The related $A L$ constraints can still be kept.
} 
between iterations. For example, in lines 7 to 9 of Algorithm 1 we recompute the set AM, possibly erasing some of the at-most constraints. Therefore, we have to take care also of any learned lemma depending on them.

The SMT solver Yices gives the option of marking assertions as retractable. If the SMT solver does not support the deletion of assertions but supports the usage of assumptions, we can replace every retractable assertion $C$, with $a \rightarrow C$, where $a$ is an assumption. Before each call, we activate the assumptions of assertions that have not been retracted by the algorithm. Notice that assertions that do have been retracted will have a pure literal $(\bar{a})$ such that $a$ has not been activated. Therefore, the solver can safely set to false $a$ deactivating the clause. Moreover, any learned lemma on those assertions will also include $\bar{a}$. For example, Z3 and Mathsat SMT solvers do not allow to delete clauses, but they allow the use of assumptions.

\section{Experimental Results}

In this section we present an intensive experimental investigation on the PMS and WPMS industrial and crafted instances from the 2012 MaxSAT Evaluation. We provide results for our new WPM2 SMT-based MaxSAT solver, for a WPM1 [2] SMT-based MaxSAT solver, the best two solvers for each category of the 2012 MaxSAT Evaluation, and three solvers which did not participate but the authors have reported to exhibit good performance. We run our experiments on a cluster featured with $2.27 \mathrm{GHz}$ processors, memory limit of $3.9 \mathrm{~GB}$ and a timeout of 7200 seconds per instance.

The experimental results are presented in Tables 1 and 2 following the same classification criteria as in the 2012 MaxSAT Evaluation. For each solver and family of instances, we present the number of solved instances in parenthesis and the mean solving time. Solvers are ordered from left to right according to the total number of solved instances. The results for the best performing solver in each family are presented in bold. The number of instances of every family is specified in the column under the sign '\#'. Since different families may have different number of instances, we also include for each solver the mean ratio of solved instances.

Our new WPM2 algorithm is implemented on top of the Yices SMT solver (version 1.0.29). The different versions of WPM2 and corresponding implementations are named $w p m 2$ where subindexes can be ${ }_{s}$ that stands for stratified approach with diversity heuristic and ${ }_{h}$ for hardening. Regarding to how we perform the cover optimization, $l$ stands for lower bound refinement based on subsetsum, ${ }_{u}$ for upper bound refinement based on satisfying truth assignment, and $b$ for binary search. Finally, $a$ stands for optimizing all the covers and ${ }_{c}$ for optimizing only covers that contain the last unsatisfiable core.

Table 1 shows our first experiment, where we evaluate the impact of each variation on the original wpm2. By using a stratified approach with the diversity heuristic $\left(w p m 2_{s}\right)$ we solve some additional instances in all categories having the best improvement in WPMS crafted. Overall, we solve 74 more instances. 


\begin{tabular}{|c|c|c|c|c|c|c|c|c|c|c|}
\hline \multirow{12}{*}{ 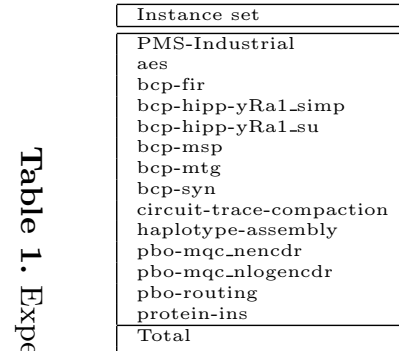 } & $\#$ & wpm 2 & $w p m 2_{s}$ & $w p m 2_{s h}$ & wpm2 shlc & $w p m 2_{s h l a}$ & $w p m 2_{s h b c}$ & $w p m 2_{s h b a}$ & wpm $2_{s h u c}$ & wpm2shua \\
\hline & 7 & $0.00(0)$ & $0.00(0)$ & $0.00(0)$ & $0.00(0)$ & $0.00(0)$ & $0.00(0)$ & $10.34(1)$ & $1836.54(1)$ & \\
\hline & 59 & $83.29(57)$ & $\begin{array}{r}23.44(57) \\
\end{array}$ & $\begin{array}{r}23.44(57) \\
\end{array}$ & $40.87(57)$ & $104.75(57)$ & $113.13(57)$ & $146.03(57)$ & $60.91(58)$ & $57.36(58)$ \\
\hline & ${ }_{38}^{17}$ & $\begin{array}{l}504.81(13) \\
380.47(19)\end{array}$ & $\begin{array}{l}155.95(12) \\
164.70(16)\end{array}$ & $\begin{array}{l}155.95(12) \\
164.70(16)\end{array}$ & $\begin{array}{r}95.16(12) \\
181.49(18)\end{array}$ & $\begin{array}{l}239.17(13) \\
667.59(19)\end{array}$ & $\begin{array}{l}151.30(13) \\
226.66(25)\end{array}$ & $\begin{array}{l}813.36(16) \\
585.82(28)\end{array}$ & $107.24(16)$ & $\begin{array}{r}160.55(16) \\
315.87(34)\end{array}$ \\
\hline & 64 & $821.88(25)$ & $756.59(26)$ & $756.59(26)$ & $283.50(28)$ & $606.35(28)$ & $283.78(31)$ & $464.47(30)$ & $711.08(36)$ & $\begin{array}{r}315.87(34) \\
912.70(34)\end{array}$ \\
\hline & 40 & $1363.97(18)$ & $852.58(23)$ & $852.58(23)$ & $1320.80(28)$ & $786.09(34)$ & $1296.44(32)$ & $940.62(37)$ & $997.38(35)$ & $578.75(39)$ \\
\hline & $\begin{array}{r}74 \\
4\end{array}$ & $\begin{array}{l}60.29(41) \\
285.74(3)\end{array}$ & $\begin{array}{r}302.54(41) \\
835.72(4)\end{array}$ & $\begin{array}{r}302.54(41) \\
835.72(4)\end{array}$ & $\begin{array}{r}296.12(41) \\
230.85(3)\end{array}$ & $\begin{array}{l}83.15(39) \\
134.79(4)\end{array}$ & $\begin{array}{r}242.78(42) \\
145.72(4)\end{array}$ & $\begin{array}{l}69.23(42) \\
151.41(4)\end{array}$ & $\begin{array}{r}103.21(43) \\
118.24(4)\end{array}$ & $\begin{array}{l}78.22(42) \\
129.10(4)\end{array}$ \\
\hline & 6 & $2.87(5)$ & $7.17(5)$ & $7.17(5)$ & $9.80(5)$ & $42.86(5)$ & $15.53(5)$ & $51.44(5)$ & $18.28(4)$ & $65.94(5)$ \\
\hline & 84 & $866.38(84)$ & $821.16(84)$ & $821.16(84)$ & $142.43(84)$ & $107.70(84)$ & $130.90(84)$ & $127.99(84)$ & $245.77(84)$ & $257.06(84)$ \\
\hline & $\begin{array}{l}84 \\
15\end{array}$ & $\begin{array}{r}362.20(84) \\
\mathbf{0 . 4 6 ( 1 5 )}\end{array}$ & $\begin{array}{r}353.14(84) \\
2.14(15)\end{array}$ & $\begin{array}{r}353.14(84) \\
2.14(15)\end{array}$ & $\begin{array}{r}24.48(84) \\
3.89(15)\end{array}$ & $\begin{array}{l}\mathbf{1 7 . 1 9 ( 8 4 )} \\
6.59(15)\end{array}$ & $\begin{array}{l}58.42(84) \\
4.88(15)\end{array}$ & $\begin{array}{l}65.79(84) \\
6.72(15)\end{array}$ & $\begin{array}{r}124.43(84) \\
5.42(15)\end{array}$ & $\begin{array}{r}140.44(84) \\
6.73(15)\end{array}$ \\
\hline & 12 & $2626.34(10)$ & $2162.13(9)$ & $2162.13(9)$ & $476.03(12)$ & $552.46(12)$ & $360.76(12)$ & $284.63(12)$ & $234.31(12)$ & $333.85(12)$ \\
\hline & 504 & $\begin{array}{r}374 \\
69.6 \%\end{array}$ & $\begin{array}{r}376 \\
70.9 \%\end{array}$ & $\begin{array}{r}376 \\
70.9 \% \\
\end{array}$ & $\begin{array}{r}387 \\
72.5 \%\end{array}$ & $\begin{array}{r}394 \\
76.0 \%\end{array}$ & $\begin{array}{r}404 \\
77.5 \%\end{array}$ & $\begin{array}{r}415 \\
81.4 \%\end{array}$ & $\begin{array}{r}425 \\
817 \%\end{array}$ & $\begin{array}{r}428 \\
836 \%\end{array}$ \\
\hline WPMS-Industrial & & & & & & & & & & \\
\hline $\begin{array}{l}\text { haplotyping-pedigrees } \\
\text { timetabling }\end{array}$ & $\begin{array}{r}100 \\
26\end{array}$ & $\begin{array}{r}16.01(22) \\
1017.27(8)\end{array}$ & $\begin{array}{r}292.29(25) \\
720.89(8)\end{array}$ & $\begin{array}{r}242.08(90) \\
651.84(8)\end{array}$ & $\begin{array}{l}154.12(92) \\
1008.59(9)\end{array}$ & $\begin{array}{l}142.59(92) \\
\mathbf{4 3 0 . 6 7 ( 9 )}\end{array}$ & $\begin{array}{l}199.61(96) \\
1544.43(9)\end{array}$ & $\begin{array}{l}86.67(95) \\
931.17(8)\end{array}$ & $\begin{array}{r}202.70(98) \\
932.77(8)\end{array}$ & $\begin{array}{r}\mathbf{1 7 6 . 0 2 ( 9 8 )} \\
1438.59(9)\end{array}$ \\
\hline upgradeability-problem & 100 & $19.19(100)$ & $19.67(100)$ & $15.27(100)$ & $96.88(100)$ & $375.70(100)$ & $99.51(100)$ & $365.70(100)$ & $97.83(100)$ & $371.96(100)$ \\
\hline Total & 226 & $\begin{array}{r}130 \\
50.9 \%\end{array}$ & $\begin{array}{r}133 \\
51.9 \%\end{array}$ & $\begin{array}{r}198 \\
73.6 \%\end{array}$ & $\begin{array}{r}201 \\
75.5 \%\end{array}$ & $\begin{array}{r}201 \\
75.5 \%\end{array}$ & $\begin{array}{r}205 \\
76.9 \%\end{array}$ & $\begin{array}{r}203 \\
75.3 \%\end{array}$ & $\begin{array}{r}206 \\
76.3 \%\end{array}$ & $\begin{array}{r}207 \\
77.5 \%\end{array}$ \\
\hline Total Industrial & 730 & 504 & 509 & 574 & 588 & 595 & 6009 & 618 & 631 & 6935 \\
\hline & & $66.1 \%$ & $67.3 \%$ & $71.4 \%$ & $73.0 \%$ & $75.9 \%$ & $77.4 \%$ & $80.2 \%$ & $80.7 \%$ & $82.5 \%$ \\
\hline $\begin{array}{l}\text { PMS-Crafted } \\
\text { frb }\end{array}$ & & & & & & & & & & \\
\hline job-shop & $\begin{array}{r}25 \\
3\end{array}$ & $68.31(3)$ & $63.37(3)$ & $\begin{array}{r}0.00(0) \\
63.37(3)\end{array}$ & $\begin{array}{r}0.0(0) \\
50.84(3)\end{array}$ & $\begin{array}{r}0.0(0) \\
53.11(3)\end{array}$ & $\begin{array}{r}0.00(0) \\
51.29(3)\end{array}$ & $\begin{array}{r}0.87(3) \\
49.87(3)\end{array}$ & $88.41(3)$ & $58.75(3)$ \\
\hline maxclicque_random & 96 & $478.65(76)$ & $471.97(76)$ & $471.97(76)$ & $435.07(77)$ & $533.97(78)$ & $423.89(83)$ & $392.04(82)$ & $565.44(87)$ & $512.17(89)$ \\
\hline maxclicque_structured & 62 & $779.71(21)$ & $592.07(21)$ & $592.07(21)$ & $511.52(22)$ & $783.37(24)$ & $650.87(25)$ & $838.38(26)$ & $802.09(26)$ & $876.90(27)$ \\
\hline $\begin{array}{l}\text { maxone_3sat } \\
\text { maxone-structured }\end{array}$ & 80 & $\begin{array}{r}16.42(80) \\
156.20(58)\end{array}$ & $\begin{array}{l}18.11(80) \\
89.15(59)\end{array}$ & $\begin{array}{l}18.11(80) \\
89.15(59)\end{array}$ & $\begin{array}{l}10.90(80) \\
14.4260)\end{array}$ & $\begin{array}{r}9.39(80) \\
32.4660)\end{array}$ & $\begin{array}{r}5.34(80) \\
13.11(60)\end{array}$ & $\begin{array}{l}\mathbf{5 . 2 9}(80) \\
46.6860)\end{array}$ & $\begin{array}{r}6.65(80) \\
\mathbf{9 . 2 4 ( 6 0 )}\end{array}$ & $6.47(80)$ \\
\hline $\begin{array}{l}\text { maxone_structured } \\
\text { min-enc_kbtree }\end{array}$ & $\begin{array}{l}60 \\
42\end{array}$ & $\begin{array}{l}1607.47(4) \\
1607.20\end{array}$ & $\begin{array}{l}89.15(59) \\
707.46(4)\end{array}$ & $\begin{array}{l}89.15(59) \\
707.46(4)\end{array}$ & $\begin{array}{l}14.42(60) \\
2057.16(5)\end{array}$ & $\begin{array}{r}32.466(60) \\
2086.38(5)\end{array}$ & $\begin{array}{l}13.1160) \\
2254.64(6)\end{array}$ & $\begin{array}{r}46.68(60) \\
1777.41(5)\end{array}$ & $\begin{array}{r}9.24(60) \\
921.09(6)\end{array}$ & $\begin{array}{r}50.43(60) \\
1906.23(5)\end{array}$ \\
\hline $\begin{array}{l}\text { pseudo_mipli } \\
\text { Thotal }\end{array}$ & $\frac{4}{372}$ & $\frac{122.23(4)}{246}$ & $\frac{93.95(4)}{247}$ & $\frac{93.95(4)}{247}$ & $\frac{109.21(4)}{251}$ & $64.35(4)$ & $27.35(4)$ & 37.81 & $54.30(4)$ & $34.23(4)$ \\
\hline Total & 372 & $\begin{array}{l}246 \\
64.9 \%\end{array}$ & $\begin{array}{r}247 \\
65.1 \%\end{array}$ & $\begin{array}{r}247 \\
65.1 \%\end{array}$ & $\begin{array}{l}251 \\
65.9 \%\end{array}$ & $\begin{array}{r}254 \\
66.5 \%\end{array}$ & $\begin{array}{r}261 \\
67.6 \%\end{array}$ & $\begin{array}{r}260 \\
67.4 \%\end{array}$ & $\begin{array}{r}266 \\
68.4 \%\end{array}$ & $\begin{array}{r}268 \\
68.5 \%\end{array}$ \\
\hline WPMS-Crafted & & & & & & & & & & \\
\hline $\begin{array}{l}\text { auc-path } \\
\text { auc-sched }\end{array}$ & $\begin{array}{l}86 \\
84\end{array}$ & $\begin{array}{l}0.00(0) \\
0.00\end{array}$ & $133.39(51)$ & $136.89(51)$ & $71.37(84)$ & $\begin{array}{r}69.59(84) \\
694\end{array}$ & $\begin{array}{r}1019.93(84) \\
2.73(84)\end{array}$ & $\begin{array}{r}948.64(5) \\
1.66(84)\end{array}$ & $\begin{array}{l}4.25(84) \\
1.71(2)\end{array}$ & $\begin{array}{r}271.167(82) \\
1.07(84)\end{array}$ \\
\hline min-enc-planning & 56 & $491.39(25)$ & $\begin{array}{r}141.80(38) \\
3.36(1)\end{array}$ & $138.29(38)$ & $3.24(56)$ & $2.47(56)$ & $0.75(56)$ & $0.77(56)$ & $0.74(56)$ & $0.80(56)$ \\
\hline $\begin{array}{l}\text { min-enc-warehouses } \\
\text { pseudo-minlib }\end{array}$ & $\begin{array}{l}18 \\
12\end{array}$ & $\begin{array}{r}11.36(1) \\
2936.02(3)\end{array}$ & $\begin{array}{r}3.36(1) \\
1533.63(3)\end{array}$ & $\begin{array}{r}3.43(1) \\
1487.40(3)\end{array}$ & $\begin{array}{r}0.07(1) \\
13.72(3)\end{array}$ & $\begin{array}{r}0.08(1) \\
11.81(3)\end{array}$ & $\begin{array}{r}0.04(1) \\
471.93(4)\end{array}$ & $0.05(1)$ & $1227.86(2)$ & $\begin{array}{r}0.05(1) \\
0.55\end{array}$ \\
\hline $\begin{array}{l}\text { pseudo-miplit } \\
\text { random-net }\end{array}$ & $\begin{array}{l}12 \\
74\end{array}$ & $\begin{array}{r}2936.02(3) \\
0.00(0)\end{array}$ & $\begin{array}{r}1533.66(3) \\
0.00(0)\end{array}$ & $\begin{array}{r}1487.00(3) \\
0.00(0)\end{array}$ & $\begin{array}{r}13.72(3) \\
1041.56(15)\end{array}$ & $\begin{array}{r}11.81(3) \\
1943.41(17)\end{array}$ & $\begin{array}{r}471.93(4) \\
1633.69(33)\end{array}$ & $\begin{array}{r}990.65(5) \\
1529.94(33)\end{array}$ & $\begin{array}{r}299.53(4) \\
3369.16(17)\end{array}$ & $\begin{array}{r}695.31(5) \\
3151.03(15)\end{array}$ \\
\hline wcsp-st & 21 & $187.40(9)$ & $310.45(10)$ & & $420.10(14)$ & & $495.17(14)$ & 470.9 & & \\
\hline $\begin{array}{l}\text { wcsp-spot5-log } \\
\text { Total }\end{array}$ & $\frac{21}{372}$ & $\frac{1067.69(8)}{46}$ & $\begin{array}{r}469.38(10) \\
114\end{array}$ & $\frac{120.60(9)}{115}$ & $\begin{array}{r}100.07(13) \\
219\end{array}$ & $\frac{59.16(13)}{221}$ & $\frac{133.75(14)}{280}$ & $\frac{48.91(13)}{281}$ & $\frac{23.84(14)}{273}$ & $\frac{16.50(14)}{271}$ \\
\hline & & $19.5 \%$ & $31.9 \%$ & $32.5 \%$ & $52.2 \%$ & $52.6 \%$ & $62.9 \%$ & $63.4 \%$ & $62.0 \%$ & $62.0 \%$ \\
\hline Total Crafted & 744 & $\begin{array}{r}292 \\
42.2 \%\end{array}$ & $\begin{array}{r}361 \\
48.5 \%\end{array}$ & $\begin{array}{r}362 \\
48.8 \%\end{array}$ & $\begin{array}{r}470 \\
59.1 \%\end{array}$ & $\begin{array}{r}475 \\
59.5 \%\end{array}$ & $\begin{array}{r}541 \\
65.2 \%\end{array}$ & $\begin{array}{r}541 \\
65.4 \%\end{array}$ & $\begin{array}{r}539 \\
65.2 \%\end{array}$ & $\begin{array}{r}539 \\
65.3 \%\end{array}$ \\
\hline Total (W)PMS & 1474 & 796 & 870 & 936 & 1058 & 1070 & 11150 & $\begin{array}{r}1159 \\
\end{array}$ & 1170 & 18174 \\
\hline & & $54.1 \%$ & $57.9 \%$ & $60.1 \%$ & $66.1 \%$ & $67.7 \%$ & $71.3 \%$ & $72.8 \%$ & $72.9 \%$ & $73.9 \%$ \\
\hline
\end{tabular}


(a) Partial Industrial

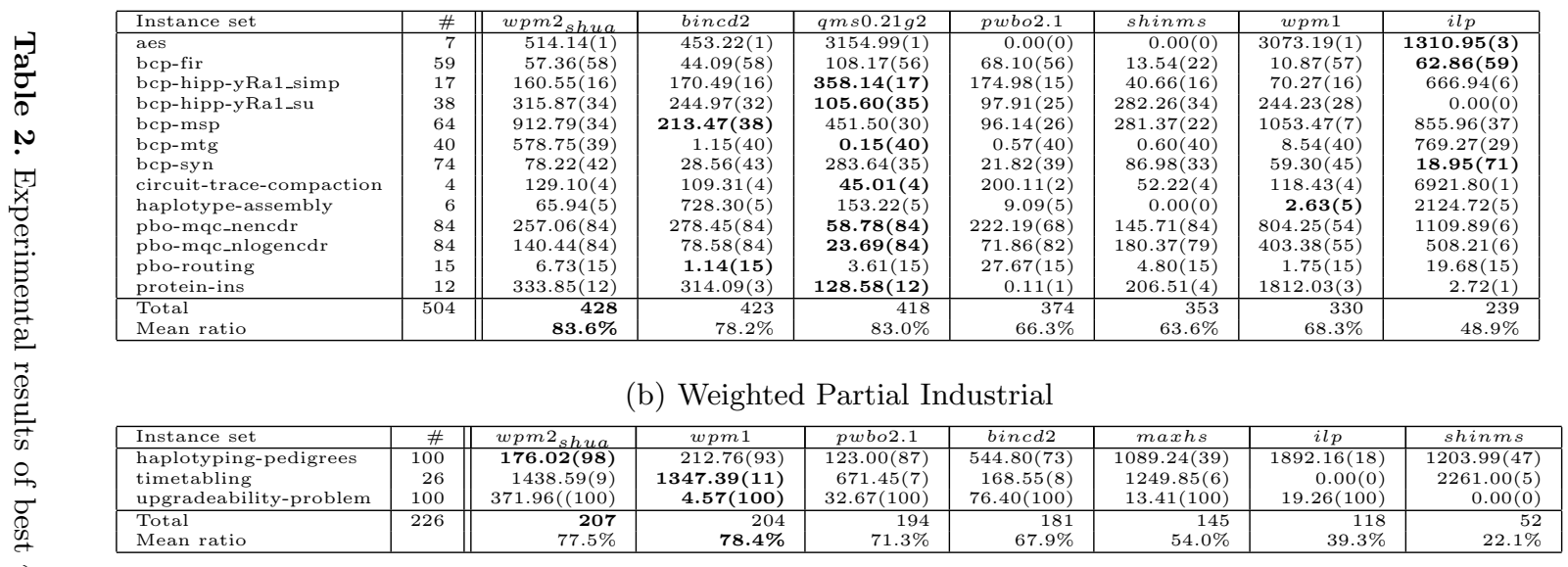

(c) Partial Crafted

\begin{tabular}{|c|c|c|c|c|c|c|c|c|c|}
\hline Instance set & $\#$ & ilp & akms_ls & $q m s 0.21$ & $\operatorname{shinms}$ & wpm2 shua & bincd 2 & $p w b o 2.1$ & wpm1 \\
\hline frb & 25 & $1152.97(13)$ & $159.47(5)$ & $346.73(25)$ & $43.52(23)$ & $\begin{array}{r}0.00(0) \\
5875(3)\end{array}$ & $0.00(0)$ & $151.16(15)$ & $0.00(0)$ \\
\hline $\begin{array}{l}\text { job-shop } \\
\text { maxclicque_random }\end{array}$ & $\begin{array}{r}3 \\
96\end{array}$ & $\begin{array}{r}0.00(0) \\
45.13(96)\end{array}$ & $\begin{array}{r}0.00(0) \\
1.09(96)\end{array}$ & $\begin{array}{r}41.51(3) \\
269.93(83)\end{array}$ & $\begin{array}{l}36.44(3) \\
339.30(76)\end{array}$ & $\begin{array}{r}58.75(3) \\
512.17(89)\end{array}$ & $\begin{array}{l}100.43(3) \\
85.29(71)\end{array}$ & $\begin{array}{r}93.17(1) \\
79.45(64)\end{array}$ & $\begin{array}{r}835.23(3) \\
652.31(59)\end{array}$ \\
\hline maxclicque_structured & 62 & $326.51(38)$ & $281.51(41)$ & $800.18(30)$ & $401.45(23)$ & $876.90(27)$ & $109.21(21)$ & $37.07(19)$ & $153.39(13)$ \\
\hline maxone_3sat & & $13.12(80)$ & $0.47(80)$ & $198.39(80)$ & $694.52(78)$ & $6.47(80)$ & $8.28(80)$ & $36.97(63)$ & $97.24(80)$ \\
\hline $\begin{array}{l}\text { maxone_structured } \\
\text { min-enckbtree }\end{array}$ & $\begin{array}{l}60 \\
42\end{array}$ & $\begin{array}{r}337.60(59) \\
\mathbf{1 6 2 . 8 9 ( 4 2 )}\end{array}$ & $\begin{array}{r}482.29(38) \\
3199.18(34)\end{array}$ & $\begin{array}{l}\mathbf{6 . 3 5}(60) \\
248.02(6)\end{array}$ & $\begin{array}{r}3.53(59) \\
513.97(5)\end{array}$ & $\begin{array}{r}50.43(60) \\
1906.23(5)\end{array}$ & $\begin{array}{l}57.42(60) \\
274.79(6)\end{array}$ & $\begin{array}{r}7.71(60) \\
306.58(2)\end{array}$ & $\begin{array}{l}718.41(42) \\
2469.35(6)\end{array}$ \\
\hline pseudo_miplib & & $34.12(4)$ & $258.91(3)$ & $1.84(4)$ & $3.44(4)$ & $34.23(4)$ & $48.74(4)$ & $93.19(4)$ & $163.61(4)$ \\
\hline Total & 372 & $\begin{array}{r}332 \\
76 \% \%\end{array}$ & 297 & $\begin{array}{r}291 \\
81 \%\end{array}$ & $\begin{array}{r}271 \\
770 \% \\
\end{array}$ & $\begin{array}{r}268 \\
68.9\end{array}$ & $\begin{array}{r}245 \\
653 \%\end{array}$ & 228 & $\begin{array}{r}207 \\
58 \%\end{array}$ \\
\hline
\end{tabular}

(d) Weighted Partial Crafted

\begin{tabular}{|c|c|c|c|c|c|c|c|c|c|}
\hline Instance set & $\#$ & & wpm 1 & $w p m 2_{s h}$ & $\operatorname{shinms}$ & $a k m s_{-} l s$ & $p w b o 2.1$ & maxhs & bincd 2 \\
\hline auc-paths & 86 & $0.49(86)$ & $108.14(63)$ & $271.16(82)$ & $317.62(84)$ & $2.57(86)$ & $110.67(19)$ & $35.41(86)$ & $1414.73(12)$ \\
\hline auc-scheduling & 84 & $0.38(84)$ & $1.56(84)$ & $1.07(84)$ & $5.81(84)$ & $68.27(84)$ & $7.65(81)$ & $965.10(78)$ & $141.77(81)$ \\
\hline min-enc-planning & $\begin{array}{l}56 \\
18\end{array}$ & $\begin{array}{l}296.52(56) \\
0.49(18)\end{array}$ & $\begin{array}{r}2.61(53) \\
78.89(17)\end{array}$ & $0.80(56)$ & $8.15(52)$ & $141.21(40)$ & $\begin{array}{l}0.46(56) \\
3.78(14)\end{array}$ & $459.18(31)$ & $32.74(54)$ \\
\hline $\begin{array}{l}\text { min-enc-warehouses } \\
\text { pseudo-miplib }\end{array}$ & $\begin{array}{l}18 \\
12\end{array}$ & $\begin{array}{l}0.49(18) \\
82.82(3)\end{array}$ & $\begin{array}{l}78.89(17) \\
157.33(4)\end{array}$ & $\begin{aligned} 0.05(1) \\
695.31(5)\end{aligned}$ & $\begin{array}{r}0.43(1) \\
127.99(5)\end{array}$ & $\begin{array}{r}20.11(2) \\
0.26(2)\end{array}$ & $\begin{array}{l}3.78(14) \\
3.97(3)\end{array}$ & $\begin{array}{l}0.18(1) \\
0.03(1)\end{array}$ & $\begin{array}{r}2.10(1) \\
1072.69(4)\end{array}$ \\
\hline $\begin{array}{l}\text { pseudo-mipib } \\
\text { random-net }\end{array}$ & 74 & $532.74(59)$ & $\begin{array}{l}4.03(70) \\
4\end{array}$ & $3151.03(15)$ & $0.00(0)$ & $4060.60(8)$ & $\begin{array}{r}32.15(35) \\
4\end{array}$ & $2770.76(10)$ & $0.00(0)$ \\
\hline wcsp-spot5-dir & 21 & $42.88(18)$ & $37.70(13)$ & $113.90(14)$ & $743.63(21)$ & $1555.46(6)$ & $61.89(8)$ & $101.11(6)$ & $127.73(12)$ \\
\hline$\frac{\text { wcsp-spot5-log }}{\text { Total }}$ & $\frac{21}{372}$ & $\frac{322.93(8)}{332}$ & $\frac{399.59(14)}{318}$ & $\frac{50(14)}{271}$ & $\frac{200.73(17)}{264}$ & $\frac{108.70(5)}{233}$ & $\frac{1.71(6)}{222}$ & $\begin{array}{l}357.32(6) \\
219 \\
\end{array}$ & $\frac{299.32(13)}{177}$ \\
\hline Mean ratio & & $78.6 \%$ & $77.4 \%$ & $62.0 \%$ & $64.8 \%$ & $45.3 \%$ & $54.4 \%$ & $41.6 \%$ & $45.6 \%$ \\
\hline
\end{tabular}




\begin{tabular}{|c|c|c|c|c|c|c|c|}
\hline solvers & $\mathrm{pms}$ & wpms & Ind. & $\mathrm{pms}$ & wpms & Cra. & Total \\
\hline wpm 2 & $\begin{array}{r}428 \\
83.6 \%\end{array}$ & $\begin{array}{r}207 \\
77.5 \%\end{array}$ & $\begin{array}{r}635 \\
82.5 \%\end{array}$ & $\begin{array}{r}268 \\
68.5 \%\end{array}$ & $\begin{array}{r}271 \\
62.0 \%\end{array}$ & $\begin{array}{r}539 \\
65.3 \%\end{array}$ & $\begin{array}{r}1174 \\
73.9 \%\end{array}$ \\
\hline wpm 1 & $\begin{array}{r}330 \\
68.3 \%\end{array}$ & $\begin{array}{r}204 \\
78.4 \%\end{array}$ & $\begin{array}{r}534 \\
70.2 \%\end{array}$ & $\begin{array}{r}207 \\
58.3 \%\end{array}$ & $\begin{array}{r}318 \\
77.4 \%\end{array}$ & $\begin{array}{r}525 \\
67.9 \%\end{array}$ & $\begin{array}{r}1059 \\
69.0 \%\end{array}$ \\
\hline bincd 2 & $\begin{array}{r}423 \\
78.2 \%\end{array}$ & $\begin{array}{r}181 \\
67.9 \%\end{array}$ & $\begin{array}{r}604 \\
76.3 \%\end{array}$ & $\begin{array}{r}245 \\
65.3 \%\end{array}$ & $\begin{array}{r}177 \\
45.6 \%\end{array}$ & $\begin{array}{r}422 \\
55.5 \%\end{array}$ & $\begin{array}{r}1026 \\
65.9 \%\end{array}$ \\
\hline$i l p$ & $\begin{array}{r}239 \\
48.9 \%\end{array}$ & $\begin{array}{r}118 \\
39.3 \%\end{array}$ & $\begin{array}{r}357 \\
47.1 \%\end{array}$ & $\begin{array}{r}\mathbf{3 3 2} \\
76.5 \%\end{array}$ & $\begin{array}{r}332 \\
78.6 \%\end{array}$ & $\begin{array}{r}664 \\
77.6 \%\end{array}$ & $\begin{array}{r}1021 \\
62.3 \%\end{array}$ \\
\hline pwbo2.1 & $\begin{array}{r}374 \\
66.3 \%\end{array}$ & $\begin{array}{r}194 \\
71.3 \%\end{array}$ & $\begin{array}{r}568 \\
67.2 \%\end{array}$ & $\begin{array}{r}228 \\
59.3 \%\end{array}$ & $\begin{array}{r}222 \\
54.5 \%\end{array}$ & $\begin{array}{r}450 \\
56.9 \%\end{array}$ & $\begin{array}{r}1018 \\
62.1 \%\end{array}$ \\
\hline shinms & $\begin{array}{r}353 \\
63.6 \%\end{array}$ & $\begin{array}{r}52 \\
22.1 \%\end{array}$ & $\begin{array}{r}405 \\
55.8 \%\end{array}$ & $\begin{array}{r}271 \\
77.0 \%\end{array}$ & $\begin{array}{r}264 \\
64.8 \%\end{array}$ & $\begin{array}{r}535 \\
70.9 \%\end{array}$ & $\begin{array}{r}940 \\
63.4 \%\end{array}$ \\
\hline$q m s$ & $\begin{array}{r}418 \\
83.0 \%\end{array}$ & & & $\begin{array}{r}291 \\
81.1 \%\end{array}$ & & & \\
\hline
\end{tabular}

Table 3. Summary of solved instances and mean ratio $\%$ for best solvers.

By adding hardening $\left(w p m 2_{s h}\right)$ we solve 66 more instances, mainly in WPMS industrial family haplotyping-pedigrees.

Regarding our three approaches for optimizing the covers, we can see that by optimizing with subsetsum $\left(w p m 2_{\text {shla }}\right)$ we solve some additional instances in all categories having the best improvement in WPMS industrial with 18 more and in WPMS crafted with 106 more. It is important to highlight that optimizing covers with subsetsum, instead of applying the subsetsum as in the original WPM2 algorithm, leads to a total improvement of 134 additional solved instances, with respect to $w p m 2_{s h}$.

Optimizing all covers by refining the upper bound $\left(w p m 2_{\text {shua }}\right)$, we get an additional boost with respect to $w p m 2_{\text {shla }}$. We can see that we solve some additional instances in all categories. We get the best improvement for PMS industrial, solving 34 additional instances, and for WPMS crafted, 50 more. Notice that the overall increase with respect to $w p m 2_{s h}$ is of 238 additional solved instances.

Binary search $\left(w p m 2_{\text {shba }}\right)$ improves 10 instances in WPMS crafted with respect to $w p m 2_{\text {shua }}$. But the global performance with respect to $w p m 2_{s h}, 223$, is not as good as only refining the upper bound (wpm $2_{\text {shua }}$ ).

Optimizing only covers that contain the last unsatisfiable core solves almost the same instances as optimizing all covers but improves the average running time in the WPMS industrial family upgradeability-problem by a factor of 4 .

Table 2 shows the results of our second experiment where we compare the best variation and implementation of our new WPM2 algorithm $\left(w p m 2_{\text {shua }}\right)$ with several solvers. In particular, we compare with the best two solvers for the PMS and WPMS industrial and crafted instances of the 2012 MaxSAT Evaluation: PMS industrial ( $q m s 0.21 g 2$, pwbo2.1), WPMS industrial (pwbo2.1 [31,32], wpm 1 [2] $\left.{ }^{8}\right)$, PMS crafted (qms0.21 [21], akms_ls [22] and WPMS crafted (wpm1, shinms [20]). We also compare with three additional MaxSAT solvers: bincd2, which is the new version of the BINCD algorithm [19] described in [33], with the best configuration reported by authors, maxhs from [14], which consists in an hybrid SAT-ILP approach, and $i l p$, which translates WPMS into ILP and applies the MIP solver IBM-CPLEX studio124 [7].

\footnotetext{
${ }^{8}$ We present in this paper a version implemented on top of the Yices SMT solver.
} 
Table 2(a) presents the results for the PMS industrial instances. Our $w p m 2_{\text {shua }}$ is the first one in solved instances with 428 and mean ratio with $93.6 \%$, closely followed by bincd 2 and $q m s 0.21 g 2$.

Table 2(b) presents the results for the WPMS industrial instances. As we can see, our wpm $2_{\text {shua }}$ and wpm 1 dominate this category with 207 and 204 solved instances and $77.5 \%$ and $78.4 \%$ mean ratio, resp.

As a summary of industrial instances, we can conclude that our wpm $2_{\text {shua }}$ is the best performing solver with a total of 635 solved instances, followed by bincd 2 with a total of 604 . We do not have results for any version of $q m s$ since it only works for PMS instances. The closest solver to the search scheme of $q m s$ would be shinms but it does not perform well for WPMS industrial.

Table 2(c) presents the results for the PMS crafted instances. The ilp approach solves 332 of 372 instances, 35 more than akms_ls. This is remarkable since branch and bound solvers, like akms_ls, have always dominated this category since 2006. PMS solver qms0.21 is the third in solved instances but the first in mean ratio with $81.1 \%$. Our wpm $2_{\text {shua }}$ is the fifth in solved instances with 268 and the fourth in mean ratio with $68.5 \%$.

Table 2(d) presents the results for the WPMS crafted instances. Again, the ilp approach is the best one, solving 332 of 372 instances, 14 more than the second one, wpm 1 . Our wpm $2_{\text {shua }}$ is the third in solved instances with 271 and the fourth in mean ratio with $62.0 \%$.

As a summary of crafted instances, we can conclude that ilp is the best performing approach, and our wpm $2_{\text {shua }}$ is the second in total solved instances.

In Table 3 we can see a summary of the solved instances and mean ratio per category for best solvers. We recall that all solvers accept weights except qms that is only for PMS. Our wpm $2_{\text {shua }}$ is the first in solved instances for both PMS industrial and WPMS industrial. In crafted categories it is the second in total solved instances. However, for both PMS crafted and WPMS crafted categories $i l p$ is the first in solved instances. We can conclude that our $w p m 2_{\text {shau }}$ is the most robust solver across all four PMS and WPMS industrial and crafted categories, followed by wpm 1 and bincd2.

\section{Conclusions and Future Work}

From the experimental evaluation, we conclude that the new WPM2 solver is the best performing solver for PMS and WPMS industrial instances and the best on the union of PMS and WPMS industrial and crafted instances. In particular, we have shown that solving to optimality the subformulas defined by covers really works in practice. As future work, we will study how to improve the interaction with the optimization of the subformulas. A portfolio that selects the most suitable optimization approach depending on the structure of the subformula seems another way of achieving additional speed-ups. Finally, we have also shown that SMT technology is an underlying efficient technology for solving the MaxSAT problem. 


\section{References}

1. Ansótegui, C., Bofill, M., Palahí, M., Suy, J., Villaret, M.: A Proposal for Solving Weighted CSPs with SMT. In: Proceedings of the 10th International Workshop on Constraint Modelling and Reformulation (ModRef 2011). pp. 5-19 (2011)

2. Ansótegui, C., Bonet, M.L., Gabàs, J., Levy, J.: Improving sat-based weighted maxsat solvers. In: Proc. of the 18th Int. Conf. on Principles and Practice of Constraint Programming (CP'12). pp. 86-101 (2012)

3. Ansótegui, C., Bonet, M.L., Levy, J.: On solving MaxSAT through SAT. In: Proc. of the 12th Int. Conf. of the Catalan Association for Artificial Intelligence (CCIA'09). pp. 284-292 (2009)

4. Ansotegui, C., Bonet, M.L., Levy, J.: Solving (weighted) partial maxsat through satisfiability testing. In: Proc. of the 12th Int. Conf. on Theory and Applications of Satisfiability Testing (SAT'09). pp. 427-440 (2009)

5. Ansotegui, C., Bonet, M.L., Levy, J.: A new algorithm for weighted partial maxsat. In: Proc. the 24th National Conference on Artificial Intelligence (AAAI'10) (2010)

6. Ansótegui, C., Bonet, M.L., Levy, J.: Sat-based maxsat algorithms. Artif. Intell. 196, 77-105 (2013)

7. Ansotegui, C., Gabas, J.: Solving maxsat with mip. In: CPAIOR (2013)

8. Bailleux, O., Boufkhad, Y., Roussel, O.: New encodings of pseudo-boolean constraints into cnf. In: SAT. pp. 181-194 (2009)

9. Barrett, C., Stump, A., Tinelli, C.: The Satisfiability Modulo Theories Library (SMT-LIB). http://www.SMT-LIB.org (2010)

10. Berre, D.L.: Sat4j, a satisfiability library for java (2006), www.sat4j.org

11. Borchers, B., Furman, J.: A two-phase exact algorithm for max-sat and weighted max-sat problems. J. Comb. Optim. 2(4), 299-306 (1998)

12. Cimatti, A., Franzén, A., Griggio, A., Sebastiani, R., Stenico, C.: Satisfiability modulo the theory of costs: Foundations and applications. In: TACAS. pp. 99-113 (2010)

13. Cormen, T.H., Leiserson, C.E., Rivest, R.L., Stein, C.: Introduction to Algorithms (3. ed.). MIT Press (2009)

14. Davies, J., Bacchus, F.: Solving maxsat by solving a sequence of simpler sat instances. In: Proc. of the 17th Int. Conf. on Principles and Practice of Constraint Programming (CP'11). pp. 225-239 (2011)

15. Eén, N., Sörensson, N.: Translating pseudo-boolean constraints into SAT. JSAT 2(1-4), 1-26 (2006)

16. Fu, Z., Malik, S.: On solving the partial max-sat problem. In: Proc. of the 9th Int. Conf. on Theory and Applications of Satisfiability Testing (SAT'06). pp. 252-265 (2006)

17. Heras, F., Larrosa, J., Oliveras, A.: MiniMaxSat: A new weighted Max-SAT solver. In: Proc. of the 10th Int. Conf. on Theory and Applications of Satisfiability Testing (SAT'07). pp. 41-55 (2007)

18. Heras, F., Larrosa, J., Oliveras, A.: Minimaxsat: An efficient weighted max-sat solver. J. Artif. Intell. Res. (JAIR) 31, 1-32 (2008)

19. Heras, F., Morgado, A., Marques-Silva, J.: Core-guided binary search algorithms for maximum satisfiability. In: Proc. the 25th National Conference on Artificial Intelligence (AAAI'11) (2011)

20. Honjyo, K., Tanjo, T.: Shinmaxsat, a Weighted Partial Max-SAT solver inspired by MiniSat+, Information Science and Technology Center, Kobe University 
21. Koshimura, M., Zhang, T., Fujita, H., Hasegawa, R.: Qmaxsat: A partial max-sat solver. JSAT 8(1/2), 95-100 (2012)

22. Kügel, A.: Improved exact solver for the weighted max-sat problem, to appear

23. Larrosa, J., Heras, F., de Givry, S.: A logical approach to efficient max-sat solving. Artif. Intell. 172(2-3), 204-233 (2008)

24. Li, C.M., Manyà, F., Mohamedou, N.O., Planes, J.: Exploiting cycle structures in Max-SAT. In: Proc. of the 12th Int. Conf. on Theory and Applications of Satisfiability Testing (SAT'09) (2009)

25. Li, C.M., Manyà, F., Planes, J.: New inference rules for Max-SAT. J. Artif. Intell. Res. (JAIR) 30, 321-359 (2007)

26. Lin, H., Su, K.: Exploiting inference rules to compute lower bounds for Max-SAT solving. In: IJCAI'07. pp. 2334-2339 (2007)

27. Lin, H., Su, K., Li, C.M.: Within-problem learning for efficient lower bound computation in Max-SAT solving. In: Proc. the 23th National Conference on Artificial Intelligence (AAAI'08). pp. 351-356 (2008)

28. Manquinho, V., Marques-Silva, J., Planes, J.: Algorithms for weighted boolean optimization. In: Proc. of the 12th Int. Conf. on Theory and Applications of Satisfiability Testing (SAT'09). pp. 495-508 (2009)

29. Manquinho, V.M., Martins, R., Lynce, I.: Improving unsatisfiability-based algorithms for boolean optimization. In: Proc. of the 13th Int. Conf. on Theory and Applications of Satisfiability Testing (SAT'10). Lecture Notes in Computer Science, vol. 6175, pp. 181-193. Springer (2010)

30. Marques-Silva, J., Argelich, J., Graça, A., Lynce, I.: Boolean lexicographic optimization: algorithms \& applications. Ann. Math. Artif. Intell. 62(3-4), 317343 (2011)

31. Martins, R., Manquinho, V.M., Lynce, I.: Exploiting cardinality encodings in parallel maximum satisfiability. In: ICTAI. pp. 313-320 (2011)

32. Martins, R., Manquinho, V.M., Lynce, I.: Clause sharing in parallel maxsat. In: LION. pp. 455-460 (2012)

33. Morgado, A., Heras, F., Marques-Silva, J.: Improvements to core-guided binary search for maxsat. In: Proc. of the 15th Int. Conf. on Theory and Applications of Satisfiability Testing (SAT'12). pp. 284-297 (2012)

34. Nieuwenhuis, R., Oliveras, A.: On sat modulo theories and optimization problems. In: SAT. pp. 156-169 (2006)

35. Sebastiani, R.: Lazy Satisfiability Modulo Theories. Journal on Satisfiability, Boolean Modeling and Computation 3(3-4), 141-224 (2007)

36. Silva, J.P.M., Sakallah, K.A.: Grasp: A search algorithm for propositional satisfiability. IEEE Trans. Computers 48(5), 506-521 (1999) 